\title{
Hashimoto's Encephalopathy: An Unusual Cause of Autoimmune Encephalopathy in a Clinically and Biochemically Euthyroid Patient
}

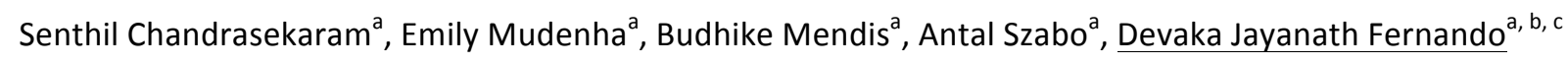

a Department of Endocrinology and Diabetes, King's Mill Hospital, Sherwood Forest Hospital NHS Foundation Trust, Sutton-in-Ashfield, United Kingdom

${ }^{b}$ Department of Diabetes and Endocrinology, University of Sheffield, Sheffield, United Kingdom

c Postgraduate Medical Centre, Sheffield Hallam University, Sheffield, United Kingdom

\begin{abstract}
Objectives: To highlight the occurrence of Hashimoto's encephalopathy - a steroid-responsive encephalopathy associated with elevated antithyroid antibodies.
\end{abstract}

Material and methods: We describe a clinically and biochemically euthyroid patient with an encephalopathy presenting with headache, mild confusion and personality changes for 6 weeks and tonic-clonic seizures upon admission

Results: There was no obvious infective or metabolic cause. The patient had a high titre of antithyroid antibodies and responded to steroid therapy.

Conclusion: This uncommon disease needs to be considered in patients presenting with neurological symptoms that remain unexplained after routine standard investigations, even when the patient is euthyroid. Early diagnosis is important, as this is a treatable condition.

Keywords: Autoimmune thyroid disease, encephalopathy, hashimotos disease

Received: $26 / 08 / 2014$

Accepted: $17 / 09 / 2014$

Published: $14 / 10 / 2014$

How to cite this article: Chandraekaram S, Mudenha E, Mendis B, Szabo A, Fernando DJ. Hashimoto's Encephalopathy: An Unusual Cause of Autoimmune Encephalopathy in a Clinically and Biochemically Euthyroid Patient, EJCRIM 2014;1:doi: 10.12890/2014_000141

Conflicts of Interests: The authors declare that they have no conflicts of interest related to this research. 


\section{Introduction}

Hashimoto's encephalopathy (HE) is a neurologic syndrome associated with Hashimoto's thyroiditis. Initially described in 1966 [1], it is thought to be autoimmune because of its association with other immunologic disorders, inflammatory findings in cerebrospinal fluid (CSF) and response to treatment with steroids [1] or an autoimmune cerebral vasculitis resulting from either endothelial inflammation or immune complex deposition [1].

\section{Case report}

This 55-year-old female patient was a smoker and a teetotaller and was not known to have diabetes or hypertension. The patient had a history of hypothyroidism; she was taking $125 \mu \mathrm{g}$ levothyroxine daily and was clinically and biochemically euthyroid. She presented with a gradually worsening constant dull frontal headache, photophobia, confusion and personality changes over the previous 6 weeks. She was treated for migraine by her family doctor with no improvement. She was disorientated and developed generalized tonic-clonic seizures. She was started on phenytoin and phenobarbitone. Her seizures resolved, but the other symptoms persisted. There was no history of fever, vomiting, loss of consciousness, drug intake, exposure to toxins or recent travel. She did not have any previous neurological deficit. She had normal bowel and bladder habits. There was no similar illness in family members.

She was confused and afebrile, with no skin rash, neck stiffness or Kernig's sign. She had word-finding difficulty, ataxic gait and right visual field visual loss. Neurological examination and systemic examination were otherwise normal.

ELISA for HIV was nonreactive as was hepatitis screening. Vasculitis screening and syphilis immunoassay were negative. CSF was negative for viral studies and showed no evidence of xanthochromia, bacterial, tuberculous or fungal infection. The computed tomography scan showed narrow triangular opacities in the left occipital and right frontal lobes, but magnetic resonance imaging (MRI) scan of the brain was normal. Electroencephalography studies showed intermittent high-amplitude slow wave activity.

The serum anti-TPO was high: $>1300 \mathrm{U} / \mathrm{ml}$ (biological reference interval of $>60 \mathrm{U} / \mathrm{ml}$ considered positive). However, the serum FT4 and TSH were normal.

A diagnosis of HE was made and the patient was started on intravenous dexamethasone. There was a rapid clinical response. She was switched to oral prednisone, starting at $1 \mathrm{mg} / \mathrm{kg}$ daily, with a slow taper. At her routine check-up after 3 weeks, she had experienced significant resolution of symptoms.

\section{Discussion}

A systematic review of HE [1] reported 85 cases and a hospital-based study estimated a frequency of 2.1/100,000 [2]. Two major patterns of presentation are described: $25 \%$ of patients follow a stroke-like pattern of multiple recurrent episodes of focal neurologic deficits with a variable degree of cognitive dysfunction and consciousness impairment [1]; the remaining $75 \%$ present with a diffuse progressive pattern of slow cognitive decline with dementia, confusion and hallucinations [1, 2]. These two clinical patterns may overlap over the course of the disease. Two-thirds of patients may experience focal or generalized tonic-clonic seizures and $12 \%$ may present with status epilepticus. Myoclonus or tremor is seen in up to $38 \%$ of patients, hyperreflexia and other pyramidal tract signs are seen in $85 \%$ of patients and psychosis, visual hallucinations and paranoid delusions have been reported in $25 \%-36 \%$ of patients [1]. 
Elevated serum levels of antithyroid antibodies remains an essential characteristic of HE [1]. The mechanism of HE does not appear to be related to the clinical functional thyroid status. In reported series, $23 \%-35 \%$ of patients were subclinically hypothyroid, $17 \%-20 \%$ hypothyroid, $7 \%$ hyperthyroid and $18 \%-45 \%$ euthyroid [1].

The pathogenic role of thyroid antibodies remains unknown. There is no evidence that any antithyroid antibody reacts with brain tissue or affects nerve function, and there is no clear correlation between the severity of the neurologic symptoms and the concentration of these antibodies [1, 2]. The resolution of symptoms with plasmapheresis supports an autoimmune aetiology [3].

In one case series, 9 of 12 patients with encephalopathy and elevated serum antithyroid antibodies had elevated CSF autoantibody titres [2]. A systematic review found that $13 \%$ of published cases of HE reported antithyroid antibodies in the CSF. However, the titres of antithyroid antibodies in the CSF do not correlate with the clinical stage of the disease, and the sensitivity and specificity of this finding remain unclear [2]. An autoantibody against the amino terminal end of the enzyme $\alpha$-enolase, an antigen of the thyroid and the brain, has been identified as a potential biomarker of Hashimoto's encephalopathy [4]. Serum autoantibody reactivity occurred in 5 of 6 patients with HE compared with 2 of 17 patients with Hashimoto's thyroiditis but no encephalopathy and in none of 25 healthy control subjects [4].

CSF analysis results were normal in our patient but a lymphocytic pleocytosis is reported in $14 \%$ of patients and may contain more than 100 cells $/ \mathrm{mm}^{3}$ in $4 \%$. An elevated protein concentration occurs in $78 \%$ of patients [1].

Non-specific EEG abnormalities are seen in $90 \%-98 \%$ patients, which is usually a non-specific slow background activity. The same pattern was observed in our patient. Focal spikes or sharp waves and transient epileptic activity are less common [5]. In a review of 82 patients with HE, brain computed tomography or MRI showed abnormalities in $49 \%$ patients, such as cerebral atrophy, focal cortical abnormality, diffuse subcortical abnormality and non-specific subcortical focal white matter abnormality.

The long-term prognosis is variable, with some patients having a progressive or a relapsing course [1]. A systematic review of 85 published cases of HE found clinical response in $98 \%$ patients treated with glucocorticoids, $92 \%$ patients treated with glucocorticoids and levothyroxine and $67 \%$ of patients treated with levothyroxine only [1].

The differential diagnosis of Hashimoto's encephalopathy must consider any condition associated with delirium, rapidly progressive dementia, seizures or focal neurologic deficits and may include stroke, transient ischaemic attack, cerebral vasculitis, carcinomatous meningitis, toxic metabolic encephalopathies, paraneoplastic syndromes, Creutzfeldt-Jakob disease, degenerative dementia and psychiatric diseases [1]. However, the possibility of another coexisting autoimmune steroid-responsive disease needs to be excluded, and patients must be kept under surveillance.

\section{Conclusion}

HE should be considered in all patients with encephalopathy of undetermined aetiology. Measuring thyroid function alone may not capture patients such as ours and the $18 \%-45 \%$ of euthyroid patients reported in the literature [1]. Measurement of thyroid antibodies should be considered in patients with encephalopathy of undetermined origin, even when clinically and biochemically euthyroid. 


\section{Learning Points}

- Hashimoto's encephalopathy is a neurologic syndrome associated with Hashimoto's thyroiditis and is thought to be autoimmune because of its association with other immunologic disorders, inflammatory findings in the cerebrospinal fluid and response to treatment with steroids or an autoimmune cerebral vasculitis resulting from either endothelial inflammation or immune complex deposition.

- The variable and non-specific clinical presentations often lead to a delay in diagnosis and initiation of treatment of Hashimoto's encephalopathy.

- Hashimoto's encephalopathy should be considered in all patients with encephalopathy of undetermined aetiology if thyroid autoimmunity coexists.

\section{References}

1. Chong JY, Rowland LP, Utiger RD. Hashimoto encephalopathy: syndrome or myth? Arch Neurol 2003;60:164-171.

2. Ferracci F, Bertiato G, Moretto G. Hashimoto's encephalopathy: epidemiologic data and pathogenetic considerations, J Neurol Sci 2004;217:165-168.

3. Nieuwenhuis L, Santens P, Vanwallaeghem P, Boon P. Subacute Hashimoto's encephalopathy, treated with plasmapheresis, Acta Neurol Belg 2004;104:80-83.

4. Fujii $\mathrm{A}$, Yoneda $\mathrm{M}$, Ito $\mathrm{T}$, Yamamura $\mathrm{O}$, Satomi $\mathrm{S}$, Higa $\mathrm{H}$ et al. Autoantibodies against the amino terminal of alpha-enolase are a useful diagnostic marker of Hashimoto's encephalopathy, J Neuroimmunol 2005;162:130136.

5. Rodrigez A, Jicha $G$, Steeves $T$, Benarroch $E$, Westmoreland B. EEG changes in a patient with steroid responsive encephalopathy associated with antibodies to thyroperoxidase (SREAT, Hashimoto's encephalopathy), J Clin Neurophysiol 2006;23:371-373. 